ISSN 1112-9867

Available online at

http://www.jfas.info

\title{
DESCRIPTION AND VARIATION FACTORS OF INDIVIDUAL CELL COUNTS OF MILK IN OF UNITS BOVINS ABOVEGROUND (TUNISIAN SAHEL)
}

\author{
Y. M'Sadak*, R. Haj Mbarek and L. Mighri \\ Institut Supérieur Agronomique de Chott-Mariem, Université de Sousse, Tunisie
}

Received: 20 March 2015 / Accepted: 26 November 2015 / Published online: 01 January 2016

\begin{abstract}
This study has as essential objective the diagnosis of breast health of a sample of 120 cattle farms conducted in above ground in the Tunisian Sahel, from the Individuals Cellular Counts (ICC). The analysis of the ICC has inferred that the infection status of the breasts is worrying ( $50 \%$ of the sample is infected). Depending on governorates, Sousse always haves the highest ICC, followed by Monastir with lower ICC and finally Mahdia with the lowest ICC. The determination and distribution of arithmetic averages (AA), geometric averages (GA), the quartiles and interquartile of ICC, led to highlight that AA of ICC are always higher than the GA of ICC. The median is closer to GA of ICC than AA of ICC, confirming the utility of the use of the GA in the cell distribution. The rank and stage of lactation, season of calving and milk production have importance on the evolution of the ICC.
\end{abstract}

Keywords: Dairy Cattle Breeding; Aboveground system; Individual Cell Count; Mammary Infection; Tunisia Semi-arid.

Author Correspondence, e-mail: msadak.youssef@yahoo.fr doi: http://dx.doi.org/10.4314/jfas.v8i1.4

\section{INTRODUCTION}

Le secteur laitier en Tunisie est l'un des secteurs stratégiques de l'agriculture et de l'économie nationale. La production laitière a évolué de 864 millions de litres en 2004 à 1218 millions de 
litres en 2014 [1]. La majorité de cette production provient de l'élevage bovin laitier. Les mammites représentent les sources les plus importantes qui occasionnent des pertes économiques en élevage bovin laitier [2, 3, 4]. Les pertes correspondent généralement au coût des traitements, aux réformes des vaches incurables, aux pertes de production laitière, aux quantités de lait rejetées [5, 6, 7]. L'avènement de méthodes de bactériologie fiables $[8,9,10]$, ainsi que d'appareils de comptage cellulaire $[9,10]$, a permis la compréhension de l'étiologie et de l'épidémiologie des mammites [11]. La bactériologie permet un diagnostic étiologique précis du microorganisme en cause [12]. Elle est considérée comme la méthode de référence, mais son coût et la technicité requise limitent son utilisation sur le terrain. Le comptage cellulaire, destiné au diagnostic de l'inflammation de la mamelle, est quant à lui peu coûteux et automatisable [13]. Il est utilisé depuis longtemps en routine comme critère de paiement du lait à la qualité $[14,15]$. Réalisé sur le lait de mélange des quatre quartiers, il permet d'identifier les vaches infectées durablement. Actuellement, des appareils de comptage cellulaire utilisables à la ferme sont disponibles. Les éleveurs peuvent évaluer rapidement l'état d'inflammation et d'infection du quartier lui-même, voire même apprécier la guérison du quartier après une mammite en se basant sur le comptage cellulaire du lait de ce quartier $[16,17]$. Généralement, le lait provenant d'une glande mammaire saine contient un certain nombre de cellules somatiques. Ces cellules sont principalement composées de globules blancs tels que les lymphocytes, les neutrophiles et les macrophages. Lorsqu'une infection survient dans la glande mammaire, les neutrophiles deviennent majoritaires et le taux cellulaire augmente fortement. Pour la vache, les cellules somatiques sont des alliés qui lui permettent de combattre une infection [18].

L'objectif de cette étude se propose de préciser, à partir de données cellulaires recueillies, l'évaluation de l'état infectieux des mamelles des vaches menées en hors sol pour explorer les solutions possibles, afin d'améliorer la qualité cellulaire du lait. A cet égard, une analyse cellulaire descriptive a été entreprise, tout en ayant recours également à l'investigation de certains facteurs responsables de l'évolution cellulaire individuelle constatée dans le lait des vaches suivies en milieu littoral semi-aride de la Tunisie. 


\section{MATÉRIEL ET MÉTHODES}

La présente étude a été réalisée sur 120 élevages bovins laitiers de trois régions du Sahel Tunisien (Figure 1), à savoir : 50 élevages de la région de Sousse, 40 élevages de la région de Monastir et 30 élevages de la région de Mahdia, totalisant 297, 185 et 225 vaches en lactation suivies respectivement.

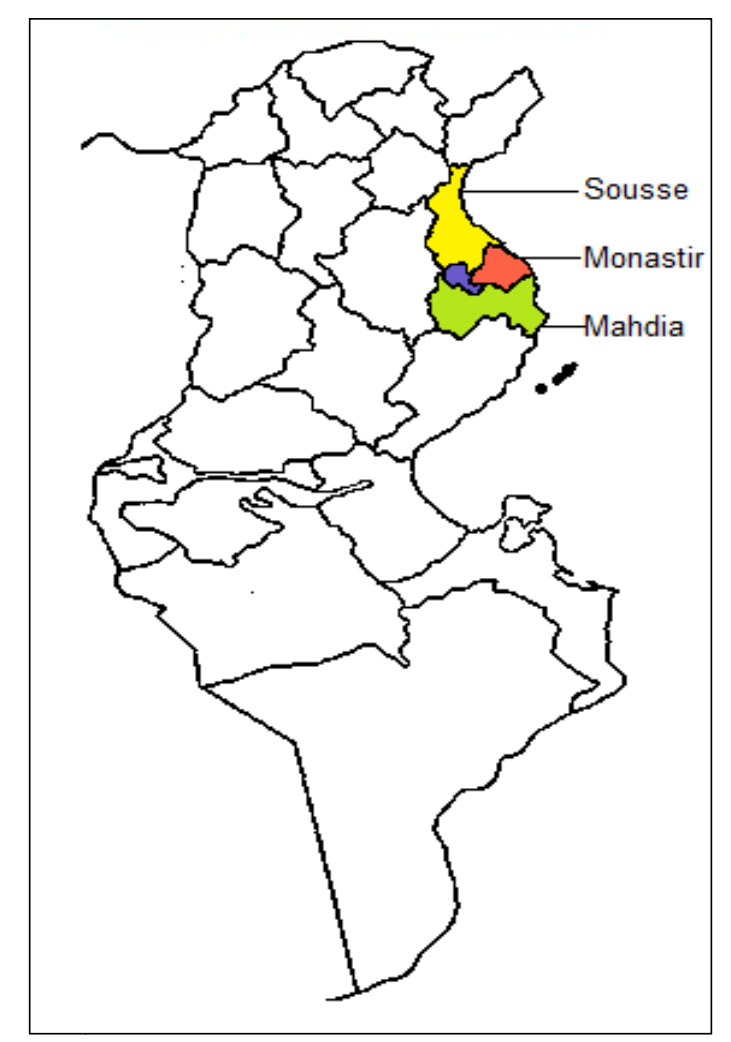

Fig.1. Localisation des régions d'étude (Sahel Tunisien)

Les vaches considérées, soumises en grande partie à la traite biquotidienne mécanique en pot, sont de la même race (Frisonne Holstein) et elles sont conduites en système hors sol, caractéristique du site d'étude. Ce système est connu par l'insuffisance, voire l'absence totale des ressources fourragères, à cause des ressources en eau généralement limitées tant quantitativement (zone à pluviométrie souvent $<350 \mathrm{~mm}$ ) que qualitativement (salinité élevée), limitant ainsi les superficies consacrées aux cultures fourragères. Le Sahel Tunisien appartient à l'étage bioclimatique semi-aride à climat généralement tempéré, influencé par l'absence de relief et par l'exposition maritime. La zone d'étude se caractérise par un hiver froid et peu pluvieux et un été chaud et sec. Les précipitations annuelles varient entre 280 et $400 \mathrm{~mm}$, 
avec une moyenne sur 10 ans de $321 \mathrm{~mm}$.

Chaque échantillon de vaches suivies, parmi les trois considérés, a fait l'objet d'une étude indépendante dans sa région. L'évaluation de la santé mammaire à travers l'analyse des comptages cellulaires du lait était l'objectif commun aux trois investigations régionales entreprises chez des petits et moyens éleveurs.

Les données des Comptages Cellulaires Individuels (CCI) ont été relevées à partir des fiches des résultats du contrôle laitier disponibles dans la base de données de l'Office d'Élevage et des Pâturages (OEP) de chaque région étudiée, en considérant huit contrôles pouvant assurer autant que possible le suivi complet d'une lactation (du vêlage au tarissement).

Les échantillons laitiers ont été prélevés périodiquement, en subissant une homogénéisation à la fin de la traite. Le lait prélevé est conservé au réfrigérateur à $4^{\circ} \mathrm{C}$ avant d'être acheminé vers le laboratoire d'analyses laitières, sans dépasser la limite de 10 jours de conservation.

Après dépouillement des données cellulaires brutes, on a calculé les moyennes arithmétiques (MA) et géométriques (MG) pour chaque contrôle par les formules suivantes : $\mathrm{MA}=\sum_{1}^{\mathrm{n}} \mathrm{CCI} / \mathrm{N} \quad$ et $\quad \mathrm{MG}=\sqrt[\mathrm{n}]{\operatorname{CCI} 1 \times \text { CCI } 2 \times \ldots \times \text { CCIn }}$

Avec CCI : Comptage Cellulaire Individuel ; N : Nombre total des échantillons de lait.

Les écarts-types, les fréquences, les quartiles (Q1, Q2 et Q3) et l'écart interquartile (Q3-Q1) ont été également déterminés.

\section{RÉSULTATS ET DISCUSSION}

\subsection{Présentation des Comptages Cellulaires Individuels}

Le CCI est un témoin de l'état inflammatoire de la mamelle et indirectement de la présence d'une infection mammaire [20, 19]. Il permet de gérer les situations d'urgence au cours de la lactation et d'assainir le troupeau pendant la période sèche [21].

Le tableau 1 révèle la répartition des CCI des trois échantillons selon la norme proposée par Fabre et al. [22]. La région de Mahdia enregistre le plus grand nombre de mamelles saines 
(62\%), suivie par la région de Monastir dont 57\% des mamelles se sont avérées saines, alors que la région de Sousse a enregistré le pourcentage le plus faible parmi les trois régions concernant les mamelles saines (55\%).

Tableau 1. Distribution des CCI selon les normes données par Fabre et al. [22]

\begin{tabular}{lllccccc}
\hline \multirow{2}{*}{$\begin{array}{c}\text { CCI } \\
(\mathbf{x 1 0 0 0} \text { cell. } / \mathbf{m L})\end{array}$} & \multirow{2}{*}{ Interprétation } & \multicolumn{2}{c}{ CCI Sousse } & \multicolumn{2}{c}{ CCI Monastir } & \multicolumn{2}{c}{ CCI Mahdia } \\
\cline { 3 - 8 } & & Effectif & $\mathbf{\%}$ & Effectif & $\mathbf{\%}$ & Effectif & $\mathbf{\%}$ \\
\hline$<300$ & Mamelle saine & 1031 & 55 & 397 & 57 & 476 & 62 \\
\hline 300 à 800 & Mamelle douteuse & 377 & 20 & 153 & 22 & 158 & 20 \\
\hline$>800$ & Mamelle infectée & 473 & 25 & 147 & 21 & 136 & 18 \\
\hline & TOTAL & 1881 & 100 & 697 & 100 & 770 & 100 \\
\hline
\end{tabular}

Le tableau 2 présente les CCI selon une autre distribution rapportée par Noireterre [21].

Tableau 2. Distribution des CCI selon les normes ramenées par Noireterre [21]

\begin{tabular}{llllcccc}
\hline \multirow{2}{*}{$\begin{array}{l}* \\
(\mathbf{x 1 0 0 0} \text { cell. } / \mathbf{m L})\end{array}$} & \multirow{2}{*}{ Interprétation } & \multicolumn{2}{l}{ CCI Sousse } & \multicolumn{2}{c}{ CCI Monastir } & \multicolumn{2}{c}{ CCI Mahdia } \\
\cline { 3 - 8 } & & Effectif & $\mathbf{\%}$ & Effectif & $\mathbf{\%}$ & Effectif & $\mathbf{\%}$ \\
\hline$\leq 200$ & Lait normal & 879 & 47 & 338 & 48 & 401 & 52 \\
\hline 200 à 500 & Mammite subclinique, Traite irritante & 355 & 19 & 148 & 21 & 172 & 22 \\
\hline 500 à 1000 & Mammite subclinique, mammite latente & 260 & 14 & 96 & 14 & 99 & 13 \\
\hline 1000 à 5000 & Doute de mammite clinique & 306 & 16 & 99 & 15 & 94 & 12 \\
\hline$>5000$ & Mammite bien établie & 81 & 4 & 16 & 2 & 4 & 1 \\
\hline & TOTAL & 1881 & 100 & 697 & 100 & 770 & 100 \\
\hline
\end{tabular}

En appliquant les règles d'appréciation (Tableau 2), on remarque que le pourcentage des vaches qui produisent un lait normal (vaches saines) diminue pour les trois régions en préservant le même ordre que précédemment. Cependant, le taux de mammite subclinique (Mamelle douteuse) augmente remarquablement en passant respectivement de 20, 22, 20\% à $33,35,34 \%$ pour les régions étudiées (Tableau 2).

Le tableau 3 relate certains paramètres statistiques décrivant les CCI relevés pour chaque contrôle réalisé par zone d'étude. Pour tous les contrôles, on remarque que la région de Sousse enregistre toujours les moyennes CCI les plus élevées suivie par la région de Monastir dont les moyennes sont moins élevées et enfin la région de Mahdia qui enregistre les moyennes les plus faibles (Tableau 3). 
Tableau 3. Répartition des CCI des contrôles considérés (x1000 cell. /mL)

\begin{tabular}{lccccccccccccc}
\hline \multirow{2}{*}{ Contrôle } & \multicolumn{3}{c}{ Moyenne } & \multicolumn{3}{c}{ Ecart-Type } & \multicolumn{3}{c}{ Minimum } & \multicolumn{3}{c}{ Maximum } \\
\cline { 2 - 14 } & S.* & Mo.* & Ma.* & S. & Mo. & Ma. & S. & Mo. & Ma. & S. & Mo. & Ma. \\
\hline $\mathbf{1}$ & 779 & 697 & 476 & 1561 & 1193 & 867 & 9 & 7 & 7 & 11397 & 6896 & 5180 \\
\hline $\mathbf{2}$ & 711 & 579 & 378 & 1787 & 1251 & 762 & 6 & 3 & 7 & 16538 & 7645 & 7028 \\
\hline $\mathbf{3}$ & 867 & 616 & 436 & 2200 & 1228 & 687 & 3 & 13 & 10 & 20646 & 7874 & 3744 \\
\hline $\mathbf{4}$ & 743 & 694 & 465 & 1471 & 1118 & 804 & 13 & 2 & 10 & 11871 & 5373 & 7141 \\
\hline $\mathbf{5}$ & 1128 & 613 & 484 & 2696 & 1095 & 453 & 13 & 30 & 56 & 18151 & 5617 & 2748 \\
\hline $\mathbf{6}$ & 1258 & 617 & 560 & 3069 & 788 & 1282 & 6 & 28 & 14 & 25071 & 3587 & 9686 \\
\hline $\mathbf{7}$ & 1135 & 600 & 596 & 2358 & 790 & 664 & 9 & 15 & 19 & 16770 & 3395 & 2836 \\
\hline $\mathbf{8}$ & 1076 & 780 & 632 & 2602 & 1125 & 982 & 12 & 15 & 44 & 21491 & 5294 & 3923 \\
\hline
\end{tabular}

* S : Sousse ; Mo : Monastir ; Ma : Mahdia

\subsection{Moyennes arithmétiques et moyennes géométriques des CCI}

Le tableau 4 illustre, pour chaque contrôle réalisé par région d'étude, les résultats cellulaires moyens obtenus en déterminant tant la moyenne arithmétique que la moyenne géométrique.

Tableau 4. MA CCI et MG CCI pour les contrôles considérés (x1000 cell. /mL)

\begin{tabular}{ccccccc}
\hline \multirow{2}{*}{ Contrôle } & \multicolumn{2}{c}{ Sousse } & \multicolumn{2}{c}{ Monastir } & \multicolumn{2}{c}{ Mahdia } \\
\cline { 2 - 7 } & MA & MG & MA & MG & MA & MG \\
\hline $\mathbf{1}$ & 779 & 320 & 697 & 220 & 476 & 162 \\
\hline $\mathbf{2}$ & 711 & 226 & 579 & 155 & 378 & 150 \\
\hline $\mathbf{3}$ & 867 & 423 & 616 & 207 & 436 & 173 \\
\hline $\mathbf{4}$ & 743 & 338 & 694 & 225 & 465 & 216 \\
\hline $\mathbf{5}$ & 1128 & 589 & 613 & 239 & 484 & 342 \\
\hline $\mathbf{6}$ & 1258 & 618 & 617 & 281 & 560 & 221 \\
\hline $\mathbf{7}$ & 1135 & 576 & 600 & 257 & 596 & 350 \\
\hline $\mathbf{8}$ & 1076 & 528 & 780 & 320 & 632 & 320 \\
\hline
\end{tabular}

Les valeurs les plus faibles de ces moyennes sont enregistrées chez les vaches de la région de Mahdia, alors que les plus élevées sont enregistrées chez les vaches de la région de Sousse. Signalons que l'hétérogénéité des résultats acquis est marquée pour les trois régions d'étude (Ecart-type élevé, dépassant la moyenne, d'où un coefficient de variation supérieur à 100\%). La moyenne CCI dépasse largement le taux de 500000 cell. /mL pour chacune des régions indiquant, ainsi, que les infections mammaires subcliniques sont répandues dans ces élevages.

\subsection{Diagramme des quartiles des CCI}


Le tableau 5 relate le diagramme cellulaire des quartiles par région d'étude et pour toute la zone du Sahel Tunisien. Le diagramme des quartiles de la distribution des CCI (Tableau 5) montre que $75 \%$ des échantillons (Q3) ont des CCI $<809000$ cell. $/ \mathrm{mL}, 624000$ cell. $/ \mathrm{mL}$ et 510000 cell. $/ \mathrm{mL}$ respectivement à Sousse, Monastir et Mahdia.

$25 \%$ des échantillons (Q1) ont des CCI $<66000$ cell. $/ \mathrm{mL}, 64000$ cell. $/ \mathrm{mL}$ et 80000 cell. $/ \mathrm{mL}$ respectivement à Sousse, Monastir et Mahdia (Tableau 5). Les valeurs observées de l'écart-type ainsi que de l'écart interquartile (Q3-Q1) sont élevées (Tableaux 3 et 5), ce qui confirme, en grande partie, l'hétérogénéité marquée des CCI des échantillons considérés. La médiane (Q2) de cette distribution est de 234000 cell. $/ \mathrm{mL}$ à Sousse, 213000 cell. $/ \mathrm{mL}$ à Monastir et 190000 cell. /mL à Mahdia, dont 50\% des échantillons ont des CCI inférieurs à ces valeurs médianes. Cette médiane est largement inférieure aux moyennes de chaque région (962000 cell. $/ \mathrm{mL}, 650000$ cell. $/ \mathrm{mL}$ et 578000 cell. $/ \mathrm{mL}$ ), ce qui réaffirme davantage le problème d'hétérogénéité des CCI dans le contexte d'étude (Tableau 5).

Tableau 5. Distribution des quartiles des CCI (x1000 cell. $/ \mathrm{mL})$

\begin{tabular}{lccc}
\hline \multicolumn{1}{c}{ Variables } & CCI Sousse & CCI Monastir & CCI Mahdia \\
\hline Q1 & 66 & 64 & 80 \\
\hline Q2 (Médiane) & 234 & 213 & 190 \\
\hline Q3 & 809 & 624 & 510 \\
\hline Q3-Q1 (Ecart interquartile) & 743 & 560 & 430 \\
\hline
\end{tabular}

\subsection{Facteurs de variation des CCI}

Compte tenu de l'importance des mammites dans les troupeaux bovins laitiers et de leurs conséquences sur les performances de production [5, 6], la qualité du lait [23, 24] et le revenu de l'éleveur [25], il est important de bien connaitre l'ensemble des facteurs de variation de la numération cellulaire du lait [26, 27].

\subsubsection{Répartition des CCI en fonction du rang de lactation}

Les CCI augmentent avec le rang de lactation [28, 29] et donc avec l'âge de l'animal [30].

Le tableau 6 révèle la répartition des vaches en fonction du rang de lactation pour montrer en moyenne l'âge des vaches considérées dans chaque région. On remarque que les primipares représentent $21 \%$ des vaches à Sousse et $32 \%$ des vaches à Monastir et à Mahdia (Tableau 6). 
Tableau 6. Répartition des vaches en fonction du rang de lactation

\begin{tabular}{ccccccc}
\hline Zone & \multicolumn{2}{c}{ Sousse } & \multicolumn{2}{c}{ Monastir } & \multicolumn{2}{c}{ Mahdia } \\
\hline Rang de lactation & Effectif & $\mathbf{\%}$ & Effectif & $\mathbf{\%}$ & Effectif & $\mathbf{\%}$ \\
\hline $\mathbf{1}$ & 63 & $\mathbf{2 1}$ & 58 & $\mathbf{3 2}$ & 73 & $\mathbf{3 2}$ \\
\hline $\mathbf{2}$ & 60 & $\mathbf{2 0}$ & 56 & $\mathbf{3 0}$ & 55 & $\mathbf{2 5}$ \\
\hline $\mathbf{3}$ & 79 & $\mathbf{2 7}$ & 21 & $\mathbf{1 1}$ & 45 & $\mathbf{2 0}$ \\
\hline $\mathbf{4}$ & 47 & $\mathbf{1 6}$ & 25 & $\mathbf{1 4}$ & 17 & $\mathbf{8}$ \\
\hline $\mathbf{5}$ & 31 & $\mathbf{1 0}$ & 15 & $\mathbf{8}$ & 14 & $\mathbf{6}$ \\
\hline $\mathbf{6}$ & 3 & $\mathbf{1}$ & 6 & $\mathbf{3}$ & 12 & $\mathbf{5}$ \\
\hline $\mathbf{6}$ & 14 & $\mathbf{5}$ & 4 & $\mathbf{2}$ & 9 & $\mathbf{4}$ \\
\hline Total & 297 & $\mathbf{1 0 0}$ & 185 & $\mathbf{1 0 0}$ & 225 & $\mathbf{1 0 0}$ \\
\hline
\end{tabular}

Le tableau 7 relate les moyennes des CCI en fonction du rang de lactation des vaches étudiées pour chaque région. Les moyennes des CCI des primipares sont toujours plus faibles que celles des multipares. Les primipares de Mahdia enregistrent les moyennes les plus faibles en comparant avec les moyennes des primipares de deux autres régions (Tableau 7). De même, les multipares de Mahdia possèdent les moyennes des CCI les plus faibles, voire plus faibles que les moyennes des primipares de Sousse.

Tableau 7. Variation des CCI (x1000 cell. $/ \mathrm{mL})$ en fonction du rang de lactation

\begin{tabular}{cccc}
\hline Zone & Sousse & Monastir & Mahdia \\
\hline Primipares & 639 & 452 & 402 \\
\hline Multipares & 1005 & 745 & 513 \\
\hline
\end{tabular}

\subsubsection{Répartition des CCI en fonction du stade de lactation}

Le nombre de cellules somatiques dans le lait augmente également au cours des stades de lactation. Les CCI au début de chaque lactation sont généralement élevés. Cette élévation est liée à la reprise de l'activité mammaire, après une phase de repos (tarissement), par la production de quantité de lait souvent faible dans les premiers jours, au cours desquels les cellules sont concentrées dans le lait produit [29]. Au cours de la lactation d'une vache saine, les CCI diminuent rapidement. Vers la fin de la lactation, les CCI augmentent à nouveau, mais progressivement. Il s'agit, comme au début, du même phénomène de concentration des cellules dans un faible volume de lait, suite à la diminution de la production. Pour des vaches infectées, les CCI restent élevés tout au long de la lactation et ne diminuent que légèrement 
par dilution vers le pic de lactation pour certaines vaches.

Le tableau 8 présente les moyennes des CCI en fonction des stades de lactation. Il est remarquable que les moyennes des CCI pour les trois régions augmentent progressivement au cours de la lactation. Ce résultat est conforme aux résultats de plusieurs études ayant dévoilé la tendance à l'augmentation des CCI avec le stade de lactation des vaches [31, 32, 33, 19, 34]. A chaque stade de lactation, les moyennes des CCI enregistrées à Sousse sont les plus élevées, alors que les plus faibles sont à Mahdia.

Tableau 8. Variation des CCI ( $\times 1000$ cell. $/ \mathrm{mL})$ en fonction du stade de lactation

\begin{tabular}{llll}
\hline Région & $\mathbf{< 1 0 0} \mathbf{j}$ & $\mathbf{1 0 0 - 2 0 0} \mathbf{j}$ & $>\mathbf{2 0 0} \mathbf{j}$ \\
\hline Sousse & $786 \pm 1862$ & $1056 \pm 2465$ & $1272 \pm 2816$ \\
\hline Monastir & $568 \pm 1451$ & $633 \pm 991$ & $705 \pm 1055$ \\
\hline Mahdia & $428 \pm 775$ & $497 \pm 864$ & $627 \pm 786$ \\
\hline
\end{tabular}

\subsubsection{Répartition des CCI en fonction de la saison de vêlage}

Les auteurs divergent concernant la saison et les mois de vêlage défavorables à la conduite sanitaire mammaire. Une étude réalisée par Rupp et al. [19] a montré que les CCI sont généralement plus élevés pour les vaches qui vêlent en printemps ou en été que pour les vaches qui vêlent en automne ou en hiver. Le tableau 9 présente les moyennes des CCI des vaches en fonction de la saison de vêlage sachant que ce paramètre n'a été évalué que dans les régions de Sousse et de Mahdia.

Tableau 9. Répartition des CCI (x1000 cell./mL) en fonction de la saison de vêlage

\begin{tabular}{lllllll}
\hline Région & \multicolumn{3}{c}{ Sousse } & \multicolumn{3}{c}{ Mahdia } \\
\hline Saison de vêlage & Effectif & $\%$ & CCI moyen & Effectif & \% & CCI moyen \\
\hline Printemps & 79 & 27 & $878 \pm 1310$ & 50 & 22 & $494 \pm 942$ \\
\hline Été & 40 & 13 & $895 \pm 1100$ & 37 & 17 & $486 \pm 779$ \\
\hline Automne & 52 & 18 & $1092 \pm 1329$ & 57 & 25 & $445 \pm 730$ \\
\hline Hiver & 126 & 42 & $901 \pm 1275$ & 81 & 36 & $472 \pm 790$ \\
\hline Total & 297 & 10 & \multirow{2}{*}{$042 \pm 1254$} & 225 & 100 & $474 \pm 811$ \\
\hline
\end{tabular}

L'hiver est la saison qui enregistre le plus grand nombre de vêlages avec $42 \%$ à Sousse et $36 \%$ à Mahdia. A Sousse, les moyennes des CCI les plus élevées sont observées durant les saisons hivernale et automnale. Par contre, à Mahdia, les moyennes des CCI ne diffèrent que 
légèrement d'une saison à une autre (Tableau 9). La moyenne générale des CCI de Mahdia est plus faible que celle enregistrée à Sousse (respectivement 474000 et 942000 cell. $/ \mathrm{mL}$ ). Ces valeurs sont élevées par rapport à la moyenne de 232000 cell. /mL, trouvée lors de l'étude réalisée chez des grands troupeaux bovins laitiers dans une région subhumide de la Tunisie par Bouraoui et al. [34].

\subsection{4 Évolution des numérations cellulaires et de la production laitière individuelle}

Le tableau 10 indique l'évolution des productions journalières moyennes de lait produit en fonction des numérations cellulaires correspondantes le jour du contrôle laitier au cours d'une lactation pour les trois régions. Diverses études ont dévoilé qu'une augmentation des CCI s'accompagne souvent d'une diminution de la production laitière (PL) [36, 37, 38]. Cette hypothèse est confirmée par les résultats relevés dans les régions étudiées (Tableau 10).

Tableau 10. Évolution des productions laitières moyennes (L) et des CCI moyens (x 100 cell. $/ \mathrm{mL})$

\begin{tabular}{lccccccccc}
\hline \multicolumn{2}{c}{ Contrôle } & C1 & C2 & C3 & C4 & C5 & C6 & C7 & C8 \\
\hline \multirow{2}{*}{ Sousse } & PL & 21 & 21 & 20 & 24 & 17 & 17 & 16 & 15 \\
\cline { 2 - 10 } & CCI & 779 & 711 & 867 & 743 & 1128 & 1258 & 1135 & 1076 \\
\hline \multirow{2}{*}{ Monastir } & PL & 18 & 19 & 19 & 18 & 17 & 17 & 16 & 15 \\
\cline { 2 - 9 } & CCI & 697 & 579 & 616 & 694 & 613 & 617 & 600 & 780 \\
\hline Mahdia & PL & 22 & 24 & 19 & 17 & 17 & 15 & 14 & 13 \\
\cline { 2 - 9 } & CCI & 476 & 378 & 436 & 465 & 484 & 560 & 596 & 632 \\
\hline
\end{tabular}

En moyenne, la numération cellulaire a évolué au cours de la lactation de façon inverse à la production laitière. On remarque que les CCI sont élevés immédiatement après le vêlage, ils diminuent jusqu'à atteindre leur minimum avec le pic de production laitière de la vache, environ au quatrième contrôle à Sousse, au deuxième contrôle à Monastir et à Mahdia, puis, ils régénèrent leur augmentation pour atteindre le maximum quelques jours avant le tarissement de la vache.

\section{CONCLUSION}

Il résulte de cette étude, quasiment descriptive, entreprise à partir des Comptages Cellulaires Individuels (CCI) sur des petits et moyens élevages bovins laitiers hors sol (tous de race 
identique : Frisonne Holstein), répartis dans trois régions littorales semi-arides de la Tunisie, que les infections mammaires sont bien établies au sein des troupeaux mis à l'étude, quelque soit la région considérée. Les variations les plus importantes observées pour les CCI sont, entre autres, celles associées au rang et au stade de lactation, à la saison de vêlage et à la production laitière. Tenant compte que chaque variable est considérée seule et indépendamment des autres, les différences observées peuvent ne pas refléter directement les effets mentionnés précédemment, mais encore des effets associés ou confondus. Par ailleurs, certaines informations cellulaires renseignant sur le statut mammaire infectieux n'étaient pas toujours disponibles pour quelques vaches suivies. L'ensemble de ces observations conduit à privilégier des analyses statistiques plus poussées lors des investigations ultérieures.

\section{RÉFÉRENCES}

[1] . GIVLAIT. Présentation de la filière lait, Groupement Interprofessionnel des Viandes rouges et du LAIT, 2014.

$\mathrm{http}: / / \mathrm{www}$.givlait.com.tn/index.php?option=com_content\&view $=$ article \&id=56\&Itemid=80

[2]. Sérieys F. ITEB, Paris, 1995, 65 p.

[3] . Mtaallah B., Oubey Z., Hammami H. Rev. Méd. Vét., 2002, 153, 4, 251-260.

[4] . Guérin P., Guérin-Faublée V. Ecole Nationale Vétérinaire Lyon, France, 2006, 140 p.

[5] . Bartlett P.C., Van Wijk J., Wilson D.J., Green C.D., Miller G.Y., Majewski G.A., Heider L.E.J. Dairy Sci., 1991, 74, 1561-1572.

[6] . Lescourret F., Coulon J.B. J. Dairy Sci., 1994, 77, 2289-2301.

[7] . Bareille N., Djabri B., Beaudeau F., Seegers H. Actes Renc. Rech. Ruminants, 2003, 10, 285-288.

[8] . Guérin-Faublée V., Carret G, Houffschmitt P. The veterinary record, 2003, 466-471.

[9]. Gandon J.B. Comparaison entre la méthode épidémiologique et la méthode bactériologique de diagnostic lors d'une épizootie de mammites en élevage bovin, Thèse Méd. Vét., ENMV Alfort, France, 2010, 85 p.

[10] . Allain M. Étude descriptive de l'identification des bactéries du lait dans un élevage à l'aide de la bactériologie, des comptages cellulaires de tank (CCT) et des comptages cellulaires individuels (CCI), Thèse Méd. Vét., ENMV Alfort, France, 2011, 122 p.

[11] . Wattiaux M.A. Université de Wisconsin, Madison, 2005, 5p.

[12] . Baillargeon P. Pré-symposium 2004 sur la mammite, 2004, 36 p.

[13] . Grappin R., Jeunet R. Revue Le Lait, 1974, n 539-540, 627-644.

[14] . Poutrel B. Rec. Méd. Vét., 1985, 161, 497-511.

[15]. Serieys F. Institut de l'Elevage, Paris, 1992. 
[16]. Ben Dhiab H. Étude des mammites dans les petits élevages bovins de la région de Monastir, PFE INA Tunis, 2001, Tunisie, 54-75.

[17] . Billon P. Journées Nationales GTV. Tours, 29-30-31 Mai, 2004, 833-839.

[18] . Baillargeon J. Réseau Canadien de Recherche sur la Mammite Bovine, 2004, 2 p.

[19] . Rupp R., Boichard D., Bertrand C., Bazin S. Revue INRA Prod. Anim., 2000, 13 (4), 257-267.

[20] . Faye B., Dorr N., Lescourret F., Barnouin J., Chassagne M. INRA Prod. Anim., 1994, 7 (1), 55-65.

[21] . Noireterre Ph. Suivi de comptages cellulaires et d'examens bactériologiques lors de mammites cliniques chez la vache laitière, Thèse Méd. Vét. ENMV Lyon, France, 2006, 98 p.

[22] . Fabre J.M., Bazin S., Faroult B., Cail P., Berthelot X. Bulletin des GTV, 1996, (2), 13-16.

[23] . Munro G.L., Grieve P.A., Kitchen B.J. J. Dairy Tech, 1984, 39, 7-16.

[24] . Barbano D.M., Rasmussen R.R., Lynch J.M. J. Dairy Sci., 1991, 74, 369-388.

[25] . Beck H.S., Wise W.S., Dodd F.H. J. Dairy Res., 1992, 59, 449-457.

[26] . Agabriel C., Coulon J.B., Sibra C. Journal C., Hauwuy A., Ann. Zootech. 1997, 46, 13-19.

[27] . Billon P., Sauvee O., Menard J.L., Gaudin V., Actes Renc. Rech. Ruminants, 1998, 5, 305-312.

[28] . Batra T.R., Mcallister A.J. Can. J. Anim. Sci., 1984, 64, 305-312.

[29] . Coulon JB, Dauver F, Garel JP. INRA Prod. Anim. 1996, 9, 133-139.

[30] . Schukken Y. H., Buurman J., Brand A., Van Der Geer D., Grommers F J. J. Dairy Sci., 1991, 73, 1343-1350.

[31] . Barnouin J., Geromegnace N., Chassagne M., Dorr N., Sabatier P. INRA Prod. Anim., 1999, 12 (1), 39-48.

[32] . Coulon JB., Lescourret F. Renc. Rech. Ruminants, 1997, 4, 265-268.

[33]. Hanzen Ch. Université de Liège, Belgique, 2009, R22, 57 p.

[34] . Sérieys F. Rec. Méd. Vét., 1985, 161, 553-566.

[35]. Bouraoui R., Rekik B., Ben Gara A. Livestock Research for Rural Development (LRRD) 21, 2009, (12), $11 \mathrm{p}$.

[36]. Seegers H., Menard J.L., Fourichon C. Actes Renc. Rech. Ruminants, 1997, 4, 233-242.

[37]. Mezine M. Analyse descriptive des facteurs de risque liés aux mammites dans des élevages d'une clientèle des Ardennes appliquant la démarche GTV partenaire. Thèse Méd. Vét., ENMV Alfort, France, 2006, 146 p.

[38] . Jacquinet S.A. Evaluation du dépistage des mammites par la conductivité électrique du lait. Thèse Méd. Vét. Université Paul-Sabatier de Toulouse, France, 2009, 135 p.

\section{How to cite this article:}

M‘Sadak Y., Haj Mbarek R. and Mighri L. Description and variation factors of individual cell counts of milk in of units bovins aboveground (Tunisian Sahel). J. Fundam. Appl. Sci., 2016, (1), 61-72. 